\title{
Cor triatriatum sinister in a 36-Year-old male with Metabolic Syndrome: A case report in sub-Saharan Africa.
}

\author{
Ahmadou Jingi ${ }^{1}$, Liliane Mfeukeu-Kuate ${ }^{2}$, Sylvie Ndongo $^{2}$, and Edvine Guela-Wawo ${ }^{2}$ \\ ${ }^{1}$ University of Bamenda \\ ${ }^{2}$ University of Yaounde I
}

November 6, 2021

\begin{abstract}
We report the first case of isolated Cor triatriatum sinister in a 36-year-old male with metabolic syndrome in sub-Saharan Africa. It is a rare congenital heart disease that consists of a fibromuscular ring that divides the atrium into two chambers. It can mimic mitral valve stenosis.
\end{abstract}

Cor triatriatum sinister in a 36-Year-old male with Metabolic Syndrome: A case report in sub-Saharan Africa.

Ahmadou Musa Jingi 1, 2*, Liliane Mfeukeu-Kuate ${ }^{2,3}$, Sylvie Ndongo Amougou ${ }^{3}$, Edvine Wawo-Yonta ${ }^{2,}{ }^{3}$.

1. Faculty of Health Sciences. The University of Bamenda. Cameroon

2. Centre Médical d'Hippodrome. Yaounde. Cameroon.

3. Faculty of Medicine and Biomedical Sciences. The University of Yaounde 1. Cameroon

Corresponding author: Dr. Ahmadou Musa Jingi. Department of Clinical Sciences. Faculty of Health Sciences. The University of Bamenda. Cameroon. E-mail: jingiahmadoumusa@yahoo.co.uk Tel: 237677864833. ORCID: 0000-0002-5320-4053 Abstract: 46 words Keywords: 4 Body of text: 1536 words Figure: 1 Tables: 0 References: 12

Abstract :

We report the first case of isolated Cor triatriatum sinister in a 36-year-old male with metabolic syndrome in sub-Saharan Africa. It is a rare congenital heart disease that consists of a fibromuscular ring that divides the atrium into two chambers. It can mimic mitral valve stenosis.

Keywords : Cor Triatriatum; Metabolic Syndrome; Cameroon; sub-Saharan Africa.

\section{Introduction :}

Cor triatriatum is a rare congenital heart disease (CHD) that consists of a fibromuscular membrane that separates an atrium into two chambers giving the impression of having three atria [1,2]. If it involves the left atrium, it is named Cor triatriatum sinister. If it involves the right atrium, it is named Cor triatriatum Dexter. Albeit rare, it is often seen in children (account for less than 1\% of all CHD) and rarely in adults $[1,2]$. It is asymptomatic or symptomatic mimicking the presentation of mitral stenosis [2]. In a large cohort of CHD in our setting, not a single case was reported [3]. To the best of our knowledge, we report the first case of asymptomatic and isolated Cor triatriatum sinister (CTS) in a 36-year old male with metabolic syndrome in Cameroon-sub-Saharan Africa.

\section{Case history}


Mr. CES was a 36-year-old male who presented to our clinic for a routine checkup before a journey. His medical history was remarkable for hypertension since four years on twice-daily Nifedipine $20 \mathrm{mg}$ and raised blood glucose since five months on no specific treatment. Further inquiry was positive for exertional palpitation, nycturia, hypoacousia of the left ear, and nocturnal snoring. He is a teacher by profession but lives a sedentary lifestyle. Physical examination was remarkable for a resting blood pressure of 198/114 mmHg (left arm) and 194/105 mmHg (right arm) with a regular heart rate of $102 \mathrm{bpm}$. His axillary temperature was $36.7^{\circ} \mathrm{C}$. His respiratory rate was $21 \mathrm{cpm}$ with an oxygen saturation of $99 \%$ on ambient air. His body mass index was $39.1 \mathrm{~kg} / \mathrm{m}^{2}$ (Weight $114.3 \mathrm{~kg}$ and height of $171 \mathrm{~cm}$ ) with an abdominal circumference of 118 $\mathrm{cm}$. The rest of the clinical examination was remarkable for acanthosis nigricans, a short and thick neck stature. We performed complementary tests as well as cardiac ultrasounds. This was remarkable for left atrial dilation (LA volume: $42 \mathrm{ml} / \mathrm{m}^{2}$ ) with a non-stenotic fibromuscular membrane that divides the left atrium into two chambers (Figure 1). There was concentric mild left ventricular (LV) hypertrophy (LV mass index of $115 \mathrm{~g} / \mathrm{m}^{2}$ and relative wall thickness of 0.58 ) with normal LV systolic function. There was diastolic dysfunction with mildly raised filling pressure (estimated resting LV filling pressure was $17 \mathrm{mmHg}$ ). The pulmonary pressure was normal at rest. There was no other anomaly of note-The patient slept off easily during the test. The echocardiographic features were compatible with hypertensive heart disease associated with Cor triatriatum sinister. The ECG showed regular sinus rhythm at $95 \mathrm{bpm}$, LA dilation, prolonged QTc $(457 \mathrm{~ms})$, and inverted T-waves in the inferior and lateral leads. Ambulatory blood pressure (BP) assessment showed very high BP values throughout with a reverse dipping pattern. Blood tests showed diabetes mellitus (fasting blood glucose of $1.55 \mathrm{~g} / \mathrm{L}$ and HbA1c of $8.2 \%$ ), marked hypokalemia (2.8 mmol/L), hyperuricemia (84 mg/L), and dyslipidemia (TC: 2.1g/L, HDLc: $0.23 \mathrm{~g} / \mathrm{L}$, TG: $2.7 \mathrm{~g} / \mathrm{L}$, and LDLc: $1.33 \mathrm{~g} / \mathrm{L}$, TC/HDLc ratio of 9.13). The urine test showed traces of protein. The full blood count showed small-sized platelets, otherwise. A thyroid function test was pending. In all, this patient presented with an incidental diagnosis of isolated Cor Triatriatum sinister in the context of metabolic syndrome (Obesity, diabetes, hypertension, dyslipidemia, hyperuricemia) with target organ damage. His 10-year Framingham score was 16\% (moderate risk). We also suspected him of having obstructive sleep apnea syndrome based on clinical context and reverse dipping BP pattern. His treatment plan was conservative associated with the control of his vascular risk factors. We proposed further evaluation of his sleep apnea.

\section{Discussion :}

We report the case of Cor triatriatum sinister (CTS) from our setting in SSA. The reason for describing this case is because it is a rare congenital heart disease that could potentially mimic mitral stenosis amidst rheumatic heart disease (RHD). Mitral stenosis due to RHD is still endemic in our setting [4]. The literature search did not yield any reported cases of CTS in SSA. This is the first described isolated case of CTS in SSA to the best of our knowledge. Secondly, we sought to provide images as didactic materials to the scientific community. No cases of CTS have been described in a large cohort of CHD studies in Cameroon [3]. The exact pathogenesis is not known [1]. Finally, the clinical context of this patient is not uncommon in our setting but is often under-investigated and undertreated according to guidelines. It is thus a nice teaching case to improve on patient care.

The patient presented with the asymptomatic form of CTS because there were no echocardiographic arguments of flow restriction across the fibromuscular membrane. LA dilatation was thought to be secondary to hypertensive heart disease. The hypertension was thought to be secondary due to the association with marked hypokalemia suggestive of hyperaldosteronism. Other arguments for a secondary cause were his young age at initial diagnosis (about 32 years of age), severely elevated BP readings, and infra-clinical target organ damage-Long QTc interval, T-wave inversion, echocardiographic LV hypertrophy, LA dilation, and proteinuria. The reverse dipping pattern or night peaking of his BP was suggestive of sleep apnea syndrome, which appears to be highly prevalent and often goes undiagnosed in our setting. Other arguments for sleep apnea were snoring, somnolence, nycturia, short and thick neck stature. Acanthosis nigrican (blackthorns) is a dark velvety discoloration around skin folds-especially the neck and axillae. This is highly suggestive of insulin resistance or the occurrence of diabetes in this patient [5]. The proposed management plan of CTS was thus conservative associated with the control of his cardiovascular risk factors. His anti-hypertensive 
regimen included blocking the renin-angiotensin-aldosterone system (RAAS) with an ACE inhibitor and anti-aldosterone (low dose spironolactone) with monitoring of serum potassium. Calcium channel blocker (Amlodipine) was added to achieve better BP control in blacks (Dike et al) [6]. Blocking the RAAS is key strategy in patients who have hypertension associated with diabetes [7]. Blocking the RAAS also has anti-proteinuria effects and organ protection. Lipid lowering strategy was with high intensity statin such as atorvastatin at least $40 \mathrm{mg}$ daily or rosuvastatin $10 \mathrm{mg}$ daily. Metformin was the drug of choice in the management of diabetes in this patient. It has been shown to have effects beyond glucose control [8]. These include improving insulin resistance, promotes weight loss, and ameliorate fatty liver disease-a new and silent epidemic in SSA. No specific hypo-uricemiant was prescribed. We have shown that certain RAAS blockers, certain calcium channel blockers, and certain statins significantly lowered serum uric acid levels in black patients with hyperuricemia (unpublished data). This is in accordance with other reports [9]. We did not prescribe low dose aspirin to this patient for primary prevention. The benefits to this patient remained debatable. We scheduled the patient for a consultation with the ophthalmologist, the dentist, and the nutritionist. Dental health and eye health are often neglected in patients with diabetes and hypertension. Cardio-respiratory polygraphy was also planned to investigate the sleep apnea syndrome. Sleep apnea syndrome (SAS)-especially obstructive SAS- is very frequent in patients with metabolic syndrome [10]. It increases the risk of poor control of cardiovascular risk factors. It should be screened and treated in all patients with metabolic syndrome. The metabolic syndrome is the constellation of at least three of the following elements-Overweight/obesity, hypertension, diabetes, dyslipidemia-with overweight/obesity being a key factor [11]. In our practice, we tend to include hyperuricemia as part of this syndrome because it is highly prevalent this group of patients. Hyperuricemia has emerged as an independent cardiovascular risk factor beyond microcrystalline arthropathy [12].

\section{Conclusion :}

We report the first case of Cor triatriatum sinister. The diagnosis was incidental in the context of the workup of metabolic syndrome. The management plan was conservative with control of the cardiovascular risk factors.

Acknowledgment : We thank the patient for permitting us to report the case.

Conflict of interest : We have no conflict of interest to declare

Author contribution : EWY did the initial consultation; AMJ and EWY performed the cardiac ultrasound; LMK and AMJ prepared the first draft. SNA and EWY critically reviewed the manuscript. All authors approved the final draft for publication.

Ethical approval and statement : The patient gave full approval for the case to be published. The patient's identity was concealed to the best of our ability.

Informed concern : The patient provided written informed consent.

Data and availability statement : All the data (resume) concerning this case has been presented.

\section{References :}

1. Al Rumitha Al Sabri NJ, Al Kindi H, Al Senaidi KS. Cor Triatriatum Sinistrum: A rare disease with a common presentation. Sultan Qaboos Univ Med J. Sultan Qaboos University; 2021;21(2):e324.

2. Claassen H, Busch C, May MS, Schicht M, Scholz M, Schulze M, et al. Cor Triatriatum Sinistrum Combined with Changes in Atrial Septum and Right Atrium in a 60-Year-Old Woman. Medicina (Mex). Multidisciplinary Digital Publishing Institute; 2021;57(8):777.

3. Kamdem F, Kedy Koum D, Hamadou B, Yemdji M, Luma H, Doualla MS, et al. Clinical, echocardiographic, and therapeutic aspects of congenital heart diseases of children at D ouala General Hospital: A cross-sectional study in sub-S aharan A frica. Congenit Heart Dis. Wiley Online Library; 2018;13(1):113-7. 
4. Nkoke C, Dzudie A, Makoge C, Luchuo EB, Jingi AM, Kingue S. Rheumatic heart disease in the South West region of Cameroon: a hospital based echocardiographic study. BMC Res Notes. Springer; 2018;11(1):1-4.

5. BROWN J, Winkelmann RK. Acanthosis nigricans: a study of 90 cases. Medicine (Baltimore). LWW; 1968;47(1):33-52.

6. Ojji DB, Mayosi B, Francis V, Badri M, Cornelius V, Smythe W, et al. Comparison of dual therapies for lowering blood pressure in black Africans. N Engl J Med. Mass Medical Soc; 2019;380(25):2429-39.

7. Williams B, Mancia G, Spiering W, Agabiti Rosei E, Azizi M, Burnier M, et al. 2018 ESC/ESH Guidelines for the management of arterial hypertension: The Task Force for the management of arterial hypertension of the European Society of Cardiology (ESC) and the European Society of Hypertension (ESH). Eur Heart J. Oxford University Press; 2018;39(33):3021-104.

8. Goodarzi MO, Bryer-Ash M. Metformin revisited: re-evaluation of its properties and role in the pharmacopoeia of modern antidiabetic agents. Diabetes Obes Metab. Wiley Online Library; 2005;7(6):654-65.

9. Cicero AF, Fogacci F, Kuwabara M, Borghi C. Therapeutic strategies for the treatment of chronic hyperuricemia: an evidence-based update. Medicina (Mex). Multidisciplinary Digital Publishing Institute; $2021 ; 57(1): 58$.

10. Poka-Mayap V, Balkissou Adamou D, Massongo M, Voufouo Sonwa S, Alime J, Moutlen BPM, et al. Obstructive sleep apnea and hypopnea syndrome in patients admitted in a tertiary hospital in Cameroon: Prevalence and associated factors. PloS One. Public Library of Science San Francisco, CA USA; 2020;15(1):e0227778.

11. Doualla-Bija M, Batchama YL, Moutchia-Suh J, Moor VJA, Kamdem F, Lekpa FK, et al. Prevalence and characteristics of metabolic syndrome in gout patients in a hospital setting in sub-Saharan Africa. Diabetes Metab Syndr Clin Res Rev. Elsevier; 2018;12(6):1007-11.

12. Kamdem F, Doualla M-S, Lekpa FK, Temfack E, Nouga YN, Donfack OS, et al. Prevalence and factors associated with hyperuricaemia in newly diagnosed and untreated hypertensives in a sub-Saharan African setting. Arch Cardiovasc Dis. Elsevier; 2016;109(10):527-32.

\section{Legend :}

Figure 1: The top panels show the four $(4 \mathrm{C})$ and two (2C) apical chamber views. The lower panels show the zoomed left atrium (LA or OG) with the non-stenotic fibromuscular band (short arrows) that separates the LA into two chambers (LA 1 and LA2). The LA1, LA2, and the right atrium give the impression of three atria in the heart, thus the term Cor Triatriatum. 


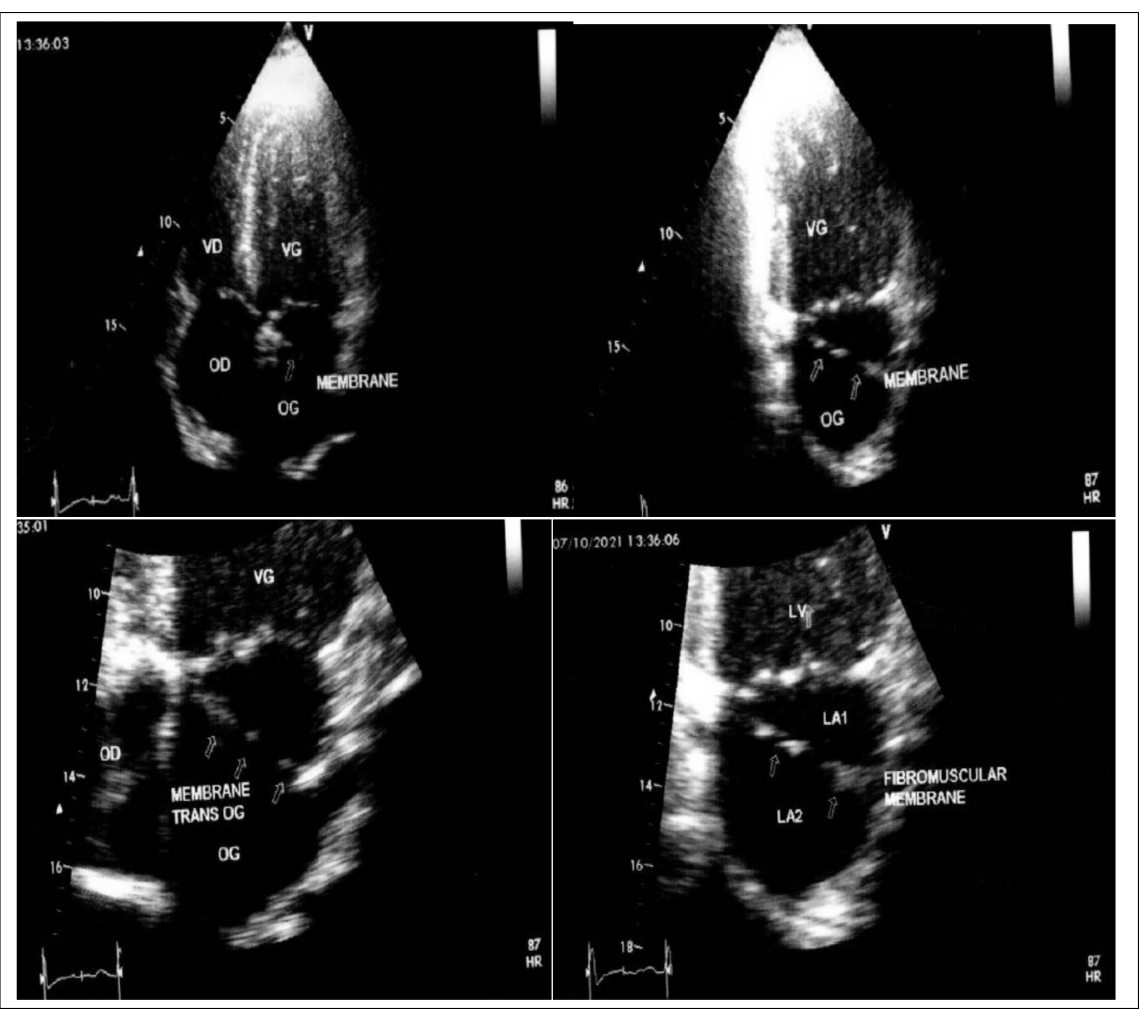

